\section{Streptococcus pneumoniae bacteremia: clinical and microbiological epidemiology in a health area of Southern Spain}

\author{
Fernando Cobo, Maria Teresa Cabezas- \\ Fernández, Maria Isabel Cabeza-Barrera \\ Department of Microbiology, APES \\ Hospital de Poniente, El Ejido, Almería, \\ Spain
}

\section{Abstract}

Streptococcus pneumoniae remains an important cause of bacteremia worldwide. Last years, a decrease of $S$. pneumoniae penicillinresistant isolates has been observed. The objective of this study was to describe the episodes of bacteremia due to $S$. pneumoniae during a period of 11 years. Epidemiological and clinical data, serotypes causing bacteremia, antibiotic susceptibility and prognosis factors were studied. Over a period of 11 years, all the episodes of $S$. pneumoniae bacteremia were analysed. Their clinical and microbiological features were recorded. Statistical analysis was carried out to determine risk factors for pneumococcal bacteremia and predictors of fatal outcome. Finally, $67 S$. pneumoniae bacteremia episodes were included in this study. The majority of cases were produced in white men in the middle age of their life. The main predisposing factors observed were smoking, antimicrobial and/or corticosteroids administration, chronic pulmonary obstructive disease and HIV infection, and the most common source of bacteremia was the low respiratory tract. The main serotypes found were 19A, 1, 14 and 7F. Seventy-seven percent of these isolates were penicillin-susceptible, and the mortality in this serie was really low. Statistical significance was observed between age, sex and race factors and the presence of bacteremia, and there was relationship between the patient's condition and the outcome. In our study, $S$. pneumoniae bacteremia is mainly from community-acquired origin mainly caused in men in the median age of the life $40 \%$ of bacteremias were caused by serotypes 19A, 1, 7F and 14. During the period of study the incidence of bacteremia was stable and the mortality rate was very low.

\section{Introduction}

Streptococcus pneumoniae is the most common cause of community-acquired pneumonia, meningitis and bacteremia in children and adults and the most common cause of acute otitis media in children. ${ }^{1}$ Overall, invasive pneumococcal disease most frequently cause infection in children less than 2 years old, older adults ( $>65$ years old) and individuals with co-morbidities and immunocompromised diseases. ${ }^{2}$

The Centers for Disease Control and Prevention (CDC) estimated approximately 15 -30 cases per 100,000 inhabitants worldwide prior to 2000 , but 160 cases per 100,000 inhabitants in children under 2 year of age and 50 83 cases per 100,000 inhabitants in adults 65 years of age and older. ${ }^{3}$ In adults, the majority of cases of pneumococcal bacteremia are associated with pneumonia, while in children the primary site of infection is frequently unknown. The overall mortality rate for pneumococcal bacteremia is $15-20 \%$ among adults, with the rate increasing to $30-40 \%$ among elderly patients.

Since introduction of the 7-valent pneumococcal conjugate vaccine (PCV7), the estimated annual incidence of invasive disease (including bacteremia) in the USA has decreased. ${ }^{4}$ However, together with reduction in invasive disease due to serotypes encompassed in PCV7, non PCV7 serotypes have emerged. ${ }^{5}$ Moreover, for several reasons, a decrease of $S$. pneumoniae penicillin-resistant strains has been observed last years worldwide. Clearly, there is a need for evaluation the new serotypes related with these episodes, the susceptibility evolution to penicillin and other antibiotics and clinical aspects to this infection. Thus, the aim of this study was to describe the episodes of bacteremia due to $S$. pneumoniae during a period of 11 years in our health area. Epidemiological and clinical data, serotypes causing bacteremia and prognosis factors were analysed.

\section{Materials and Methods}

\section{Patients}

The clinical records of all patients with positive blood cultures for $S$. pneumoniae at this hospital in the period from January 1999 to October 2010 were reviewed retrospectively. This centre is an approximately 250-bed secondary care institution, serving as reference hospital for a rural and urban area of nearly 300,000 inhabitants (28\% immigrants from 140 nationalities). In this cohort, there were 52 (77.6\%) Caucasian patients and 15 (22.4\%) black patients. The majority of immigrants come from Africa (sub-Saharian countries).

\section{Microbiology}

Two blood culture sets were usually drawn from an antecubital vein. Blood cultures were
Correspondence: Fernando Cobo, Department of Microbiology (Biotechnology Area), APES Hospital de Poniente, Ctra de Almerimar s/n, 04700 El Ejido, Almería, Spain.

Tel. +34.950.022 638 - Fax: +34.950 .022 .601 .

E-mail: fernando.cobo.sspa@juntadeandalucia.es

Key words: Streptococcus pneumoniae, bacteremia, risk factors, antibiotic susceptibility, epidemiology, serotypes.

Acknowledgements: we would like to acknowledge to Mónica Marín-Palma for her help in the statistical analysis. We also would like to acknowledge to Dra Asunción Fenoll for serotyping all the S. pneumoniae strains and to Ms Angela Helen Barnie for the English correction of this manuscript.

Contribution: MTCF, isolation, identification, serotyping of strains of $\mathrm{S}$. pneumoniae, study design, manuscript review; MICB, isolation and identification of $\mathrm{S}$. pneumoniae strains, study design, data collection, manuscript review; FC, study design, data collection and analyses, manuscript writing

Conflict of interest: the authors report no conflicts of interest.

Received for publication: 20 October 2011

Revision received: 29 February 2012.

Accepted for publication: 16 April 2012.

This work is licensed under a Creative Commons Attribution NonCommercial 3.0 License (CC BYNC 3.0).

(C) Copyright F. Cobo et al., 2012

Licensee PAGEPress, Italy

Infectious Disease Reports 2012; 4:e29

doi:10.4081/idr.2012.e29

performed with a colorimetric blood culture analyser, with FAN-TM culture bottles (BacT/ALERT 3D, BioMérieux, Durham, NC, USA) incubated routinely for 5 days. All isolates were identified to species level by a panel of biochemical tests by an automatized analyser (VITEK 2, bioMérieux) and by means of optochin susceptibility. Antimicrobial susceptibility tests were performed by the E-test technique. Penicillin G, Amoxicillin, Erythromycin, Chloramphenicol, Vancomycin, Cefotaxime and Tetracycline were tested and included in this study. The strains were sent to the Spanish National Reference Centre for Microbiology (Madrid, Spain) in order to serotyping.

\section{Clinical definitions}

Bacteremia was considered to be community-acquired when $S$. pneumoniae was isolated from blood cultures taken within 48 hours. of 
hospital admission and the patient had not been hospitalised in the previous 2 weeks. Bacteremia was defined as nosocomial if positive blood cultures were obtained after 48 hours or more since hospital admission, and if there was no evidence of pneumococcal infection at the time of hospitalization.

The patient's condition was classified according to the system of Winston et al. ${ }^{6}$ On this latter basis, the patient's condition before the bacteraemia was classified as critical (condition rapidly deteriorating with death in a short period of time not unlikely), poor (condition deteriorating with death possible but not imminent), fair (condition deteriorating but death not likely) or good (condition stable).

The following predisposing factors for bacteremia were considered: i) Splenectomy, ii) Drepanocytosis, iii) HIV infection, iv) Bone marrow transplantation, v) acute myeloid leukaemia. Secondary factors considered were the use of antibiotics and/or corticosteroids within 10 days before the episode of bacteremia, chronic pulmonary obstructive disease, smoking and alcohol abuse, diabetes mellitus, lymphoma, multiple myeloma, chronic lymphatic leukaemia, hypogammaglobulinemia and complement deficit.

Septic shock was defined as the presence of severe sepsis accompanied by a sustained decrease in systolic blood pressure $<90 \mathrm{mmHg}$ or a drop of $40 \mathrm{mmHg}$ from baseline, for at least 1 hour, despite adequate fluid resuscitation, or any such decrease that required vasoactive drugs. ${ }^{7}$

\section{Data}

Clinical histories from selected patients were revised and both epidemiological data (sex, age, predisposing factors, race ethnicity, community-acquired or nosocomial-acquired bacteremia, PCV7 vaccine administration, serotype) and clinical/analytical data (patient's condition, outcome, leukopenia and/or neutropenia presence, antibiotics susceptibility) were registered. Susceptibility categories were determined using the Clinical and Laboratory Standards Institute guidelines. ${ }^{8}$

\section{Statistical analysis}

This is a transversal and descriptive epidemiological study over a sample of 67 patients with $S$. pneumoniae bacteremia. Prevalence of predisposing factors and confidence interval $95 \%$ were calculated. The independent analysis between the different variables was performed by means of Pearson $\chi^{2}$ test for contingence tables. The determination of variables to be included in the model was based on a $\mathrm{P}$ value lower than 0.05 . The SPSS $^{\circledR}$ software package (version 10.0) was used for statistical analysis.

\section{Results}

During the period of study, 67 S. pneumoniae bacteremia episodes were finally included. There were 49 male and 18 female patients. Their mean age (standard deviation) was 49.3 years $( \pm 23)$. The majority of cases of $S$. pneumoniae bacteremia were produced in patients between 3-65 years of age, followed by those > 65 years old. Data about the distribution of bacteremias per year of study are shown in Figure 1. Seventy percent of patients had predisposing factors (Table 1), mainly smoking, followed by antimicrobial/corticosteroids administration, chronic pulmonary obstructive disease, diabetes mellitus and HIV infection. A primary source of bacteremia was identified in 47 episodes, as confirmed either by culture or by clinical evidence of a primary focus. The most common focus was the low respiratory tract recorded for 46 patients and cerebrospinal fluid for 1 patient. The source of bacteremia was considered unknown in 20 episodes. Seventy-three percent of bacteremia episodes were produced in male patients, being more frequent in white/Caucasian race (77.6\%); 100\% of episodes were from community-acquired origin. With respect to the serotypes found, Figure 2 shows the total distribution. Serotype 19A was the most frequent, followed by serotypes $1,7 \mathrm{~F}$ and 14 . With respect to susceptibility to penicillin, $77.6 \%$ of the isolates were penicillin-susceptible, while $17.9 \%$ were penicillin-intermediate and only $4.5 \%$ of these isolates were penicillin-resistant. Among the 67 strains isolated, the susceptibilities to other different antimicrobials (cefotaxime, amoxicillin, erythromycin, chloramphenicol, tetracycline and vancomycin) were determined. The results are shown in Figure 3. The relationship between the age, sex and ethnicity features and the presence of bacteremia was analysed (confidence interval 95\%). Statistical significance was observed because white/Caucasian men $>65$ years or older had higher probability to have $S$. pneumoniae bacteremia $(\mathrm{P}<0.045)$.

Among the 67 cases of bacteremia, the outcome was satisfactory in 63 and only in 4 cases the outcome was fatal. These cases were produced by the serotypes $17,19 \mathrm{~A}, 19 \mathrm{~F}$ and 8 but no statistical significance was observed when mortality related with serotype were compared $(\mathrm{P}=0.223)$. When serotype and susceptibility to penicillin were compared, no statistical significance was observed, but it would be aware with serotypes 14 and $19 \mathrm{~A}$ because it was observed higher resistance to antimicrobials than in the rest of serotypes $(\mathrm{P}<0.453)$. There was a relationship between the patient's condition and the outcome. Clinical condition critical and poor and the presence of septic shock are related with all cases of mortality, so the
Table 1. Predisposing factors of Strepto coccus pneumoniae bacteremia (67 episodes).

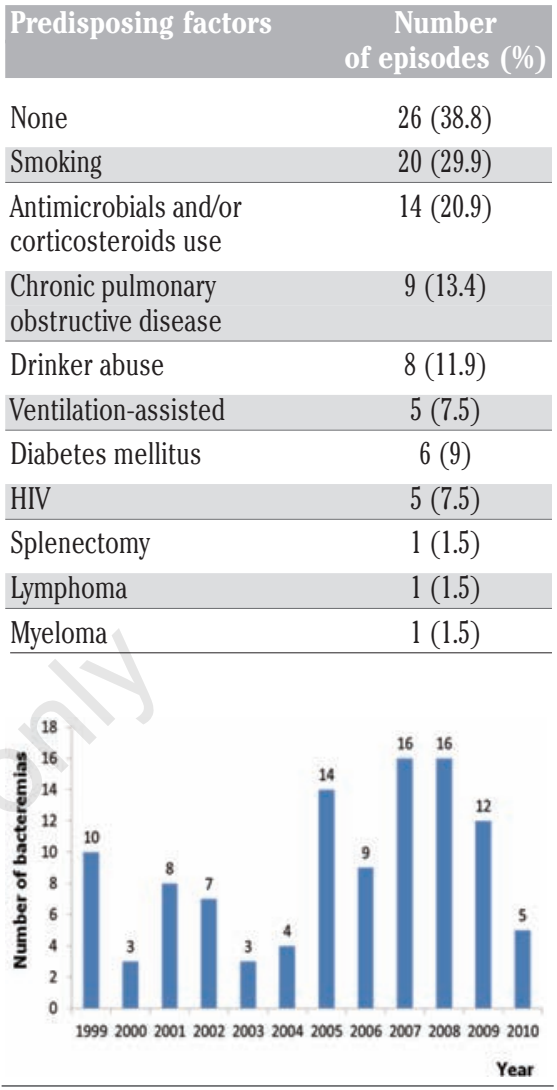

Figure 1. Number of bacteremias caused by Streptococcus pneumoniae during the period of study.

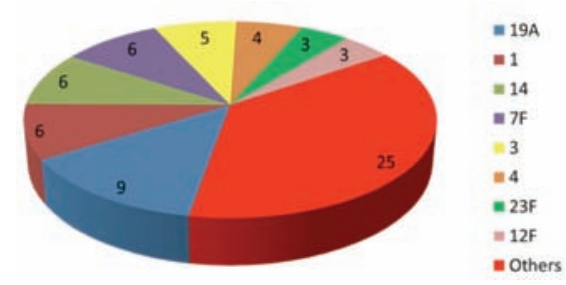

Figure 2. Main serotypes of 67 Streptococcus pneumoniae bacteremia episodes.

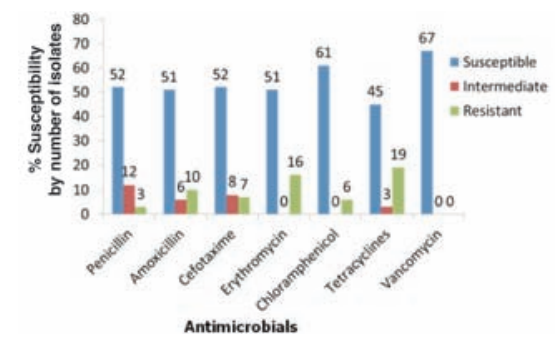

Figure 3. Susceptibility to different antimicrobials among the 67 episodes of Streptococcus pneumoniae bacteremia. 
patients in this condition had higher probability of death $(\mathrm{P}<0.002)$.

\section{Discussion}

The present study examined a large cohort of patients with $S$. pneumoniae bacteremia. Five variables were significantly associated with mortality from $S$. pneumoniae bacteremia: age $>65$ years, male sex, Caucasian race, clinical condition critical and poor and septic shock. The incidence of S. pneumoniae bacteremia is affected by geographic location, time of year, age and vaccination status. The incidence of this kind of bacteremia is 15-35 cases per 100,000 inhabitants, ${ }^{9}$ and there are differences by groups of age.$^{10}$ In our study the median annual incidence was 2.9 cases/ 100,000 inhabitants, being homogeneous during the period of study (range 1-5.3/100,000 inhabitants). However, it is possible that these cases are being underestimated because the majority of respiratory diseases accompanied with febrile syndrome are being treated with antibiotics without obtaining blood cultures. In this cohort of patients, there was no administration of the vaccine in the majority of our patients (only in a child the vaccine was administered). However, there was an episode of bacteremia caused by the serotype 7F (no included in the heptavalent vaccine) in a patient who received this vaccine. This fact emphasizes the need to be aware for the possibility of increase in non-PCV7 serotype incidence infections, while PCV23 vaccine is being introduced and applied in the general population. The present findings show that serotypes $19 \mathrm{~A}, 1,7 \mathrm{~F}$ and 14 are the most common serotypes among $\mathrm{S}$. pneumoniae bacteremia in our health area. McKensie et al. ${ }^{11}$ found high levels of serotype 14, while Hausdorff et al. ${ }^{12}$ showed that, in all age groups, serotypes 1, 9 and 14 were most frequently isolates from blood cultures in Europe. Thus, the distribution of serotypes differs among geographical regions. ${ }^{13}$

In our study, the majority of cases of bacteremia caused by this microorganism were produced in patients between 3-65 years followed by $>65$ years old. This age factor has been found in most studies. Metlag et al. ${ }^{14}$ noted a higher level of bacteremia among patients over the age of 65 . Other authors ${ }^{15}$ have also found a higher level of bacteremia among patients below the age of 5 years. However, in our study, the number of bacteremia episodes in patients below the age of 3 years was low (9 patients, 13.4\%).

Pneumococcal bacteremia is produced frequently in individuals with predisposing factors. The incidence of bacteremia and invasive infection is high in immunocompetent patients with co-morbidities such as alcohol abuse, diabetes mellitus, asthma, recent influenza infection and exposure to cigarette smoke. ${ }^{1}$ Moreover, the risk of bacteremia and invasive infection is increased among patients with primary or acquired immunodeficiencies (e.g HIV infection, haematological diseases, splenectomy, organ transplant recipients). ${ }^{1}$ In our work, sixty-one percent of patients had 1 or more risk factors. The main predisposing factor for $S$. pneumoniae bacteremia was cigarette smoking (29.9\%), use of antimicrobials and/or corticosteroids previously (20.9\%), presence of chronic pulmonary obstructive disease (13.4\%), alcohol abuse (11.9\%), diabetes mellitus (9\%) and HIV infection (7.5\%). Other risk factors detected were the presence of lymphoma or myeloma (2 cases) and splenectomy (1 case). Like in other studies, ${ }^{11}$ the main source of bacteremia in our patients was the low respiratory tract in 46 (68.6\%). In one case only the source of infection was the cerebrospinal fluid and in 20 patients (29.8\%) the origin could not be found. On the other hand, $22.3 \%$ of $S$. pneumoniae isolates were penicillin-nonsusceptible ( $4.4 \%$ resistant) in the present study. In our country, the percentage of invasive strains penicillin-nonsusceptible in the general population range from $19-45 \%,{ }^{16}$ similar to our results. Other studies, have also shown similar results with respect to levels of penicillin-nonsusceptible strains. ${ }^{17}$ With respect to the rest of antimicrobials tested, the results are shown in Table 2.

In our study, the mortality associated to bacteremia was $6 \%$. This rate is lowest than those described in other studies. ${ }^{11,17,18}$ However, our study demonstrated that susceptibility to penicillin is not a predicting factor for mortality $(\mathrm{P}=0.217)$, although this fact could be due to the low number of patients death in this cohort and the susceptibility to penicillin of the most strains. In conclusion, in our health area characterized with a high level of immigrant population, $S$. pneumoniae bacteremia is from community-origin, mainly caused in white/ Caucasian men in the median age of life or $>65$ years old. Forty percent of bacteremias were caused by serotypes $19 \mathrm{~A}, 1,7 \mathrm{~F}$ and 14 , and during the period of study the incidence of disease has not decreased, probably due to the no administration of PCV7 anti-pneumococcal vaccine in the majority of our patients. In our opinion, although the benefits of vaccine have been demonstrated, there are data that suggest the underutilization of this vaccine, such as in our health area. Thus, health workers should be aware to this fact in order to recommend the use of the vaccine in much more patients and in order to suspect this infection in the general population. Finally, the presence of septic shock and clinical situation critical and poor were some factors associated to higher mortality in these kinds of patients.

\section{References}

1. Lynch JP, Zhanel GC. Streptococcus pneumoniae: epidemiology, risk factors, and strategies for prevention. Semin Respir Crit Care Med 2009;30:189-209.

2. Naheed A, Saha SK, Breiman RF, et al. Multihospital surveillance of pneumonia burden among children aged $<5$ years hospitalized for pneumonia in Bangladesh. Clin Infect Dis 2009;48Suppl 2:S82-9.

3. Centers for Disease Control and Prevention (1997). Prevention of pneumococcal disease: recommendations of the Advisory Committee on Immunization Practices (ACIP). MMWR Recomm Rep 46 (NoRR-8), 1-24. Available from: http://www.cdc.gov/mmwr/preview/mmwrh tml/00047135.htm

4. Centers for Disease Control and Prevention (2007). Active bacterial care surveillance report, Emerging infections program Network, Streptococcus pneumoniae (2006). Available from: www.cdc.gov/ ncidod/dbmd/abcs/survreports/spneu06.pdf

5. Tyrrell GJ, Lovgren M, Chui N, et al. Serotypes and antimicrobial susceptibilities of invasive Streptococcus pneumoniae pre and post seven valent pneumococcal conjugate vaccine introduction in Alberta, Canada, 20002006. Vaccine 2009;27:3553-60.

6. Winston DJ, Murphy W, Young LS, et al. Piperacillin therapy for serious bacterial infections. Am J Med 1980;69:255-61.

7. Bone RC, Fisher CJ, Clemmer TP, et al. A controlled trial of high-dose methylprednisolone in the treatment of severe sepsis and septic shock. N Engl J Med 1987;317: 653-8.

8. Clinical and laboratory Standards Institute (2011). Performance Standards for Antimicrobial Susceptibility testing, twentyfirst information supplement, M100-S21. Clinical and Laboratory Standards Institute, Wayne, PA, USA. Available from: www. rsu.ac.th/medtech/files/CLSI\%202011.pdf

9. Fariñas-Álvarez C, Fariñas MC, GarcíaPalomo JD, et al. Prognosis factors for pneumococcal bacteremia in a university hospital. Eur J Clin Microbiol Infect Dis 2000;19:733-41.

10. Mirzanejad Y, Roman S, Talbot J, et al. Pneumococcal bacteremia in two tertiary care hospitals in Winnipeg, Canada. Chest 1996;109:173-8.

11. McKensie H, Reid N, Dijkhuizen RS. Clinical and microbiological epidemiology of Streptococcus pneumoniae bacteremia. J Med Microbiol 2000;49:361-6.

12. Harsdorff W, Bryant J, Kloek C, et al. The contribution of specific pneumococcal serogroups to different disease manifestations: implications for conjugate vaccine 
formulation and use. Clin Infect Dis 2000;30:122-40.

13. Hsieh YC, Lin PY, Chiu CH, et al. National survey of invasive pneumococcal diseases in Taiwan under partial PCV7 vaccination in 2007: emergence of serotype 19A with high invasive potential. Vaccine 2009;27: 3313-8.

14. Metlag JD, Hofman J, Cetron MS, et al. Impact of penicillin susceptibility on medical outcomes for adult patients with bac- teriemic pneumococcal pneumonia. Clin Infect Dis 2000;30:520-8.

15. Kaplan S, Mason EO, Barson WJ, et al. Three-year multicenter surveillance of systemic pneumococcal infections in children. Pediatrics 1998;102:538-45.

16. Rubio FS, Aznar R, Martin I, et al. Bacteriemia en el anciano: factores asociados y pronósticos. Rev Clin Esp 1998;198:7-10.

17. Margein J, Guillemot D, Dupont MJ, et al.
Clinical and microbiological epidemiology of Streptococcus pneumoniae bacteremia in eight French counties. Clin Microbiol Infect 2003;9:280-8.

18. Ekdahl K, Martenson A, Kamme C. Bacteraemic pneumococcal infections in southern Sweden 1981-1996: trends in incidence, mortality age-distribution, serogroups and penicillin resistance. Scand J Infect Dis 1998;30:257-62. 